\title{
Research on Cooperative Strategy of Multiple Rockets
}

\author{
Ke Yi ${ }^{1}$, Si-hong She ${ }^{1}$, Shun Zhang ${ }^{1}$, Bang-ya Hu²,a, Meng Liu \\ ${ }^{1}$ Systems Engineering Research Institute, Beijing, China \\ ${ }^{2}$ School of Aerospace Engineering, Beijing Institute of Technology, Beijing, China
}

\begin{abstract}
In order to enhance the penetration ability of the rocket and the damage degree of the target, the trajectory design and optimization of the rockets are carried out, and the cooperative strategy of multiple rockets is designed. Firstly, the particle motion model of rocket is established, and the power and guidance scheme are given. Then, a cooperative attack strategy based on different launch points for multiple rockets is proposed, which transforms the cooperative attack into the optimization of launch points. The optimization model of the launch point under the expected attack time is established, and the model is solved by the cultural algorithm. The distribution of the launch point under different attack time is obtained and fitted as curves under corresponding conditions. Finally, aiming at the problem of multiple rockets cooperative combat, using the obtained curves, three kinds of cooperative strategies which are suitable for different attack time requirements are proposed and verified by simulation.
\end{abstract}

Keywords. multiple rockets, cooperative combat, trajectory design and optimization, cultural algorithm

\section{Introduction}

With the development of anti-missile technology, more and more attention has been paid to multiple missiles cooperative attack and defense, and cooperative guidance technology has also been developed rapidly. If multiple rockets can attack the target together, the penetration ability and damage degree of the rocket can be greatly enhanced. Therefore, this paper proposes a scheme to realize the cooperative combat of multiple rockets by changing the launching point without changing the guidance parameters of rockets.

At present, scholars have done some research on missile cooperative combat. In [1] it is determined that the MRSI (multiple rounds simultaneous impact) scheme is to find out all possible trajectories for a certain range, and then select the trajectories satisfying MRSI. Reference [2] made some researches on MRSI of onedimensional correction missiles launched by naval gun, put forward a fire control algorithm to achieve MRSI by changing the launching angle and damping ring opening time, and then in [3] it is improved on the basis of the algorithm to meet the needs of fast and violent attack on the target. In order to improve the trajectory calculation accuracy of MRSI of single gun, a local interpolation algorithm is proposed based on the two-dimensional particle trajectory equation and the two-dimensional vacuum trajectory oblique range formula in [4]. Although some algorithms have been proposed by some scholars for MRSI, most of the existing researches are still on the guided shell of naval gun, and the algorithm still has the problems of long calculation time and large calculation amount. A guidance problem for impact time control applicable to salvo attacks is considered based on the sliding mode control in [5]. Time constraints are achieved by designing an impact-time vector guidance law in [6]. Reference [7] suggested a "Close-Formation Approach" attack tactics as a new tactics to operate multiple antiship missiles. However, because the rockets generally does not have the ability of inter missile communication, the existing cooperative formation technology cannot be applied to the cooperative combat of rockets. At present, there is no mature and effective strategy for the research of rockets cooperative combat.

In this paper, a three degree of freedom particle trajectory model of rocket is established, and the power and guidance scheme are designed. Aiming at the cooperative combat of multiple rockets, the cooperative attack is realized by changing the launch site of rockets, and the cooperative attack is transformed into the optimization of launch point. The optimization model of cooperative attack is established. The distribution curve of launch point with the same attack time is obtained by solving the optimization model. Three cooperative strategies are proposed and verified by simulation.

\section{Modeling of rocket motion}

\subsection{Rocket motion model}

The equations of motion of the rocket's center of mass in the launch coordinate system are

\footnotetext{
a Corresponding author: 644482455@qq.com
} 


$$
\left\{\begin{array}{l}
m \frac{d V}{d t}=P \cos \alpha \cos \beta-X-m g \sin \theta \\
m V \frac{d \theta}{d t}=P\left(\sin \alpha \cos \gamma_{V}+\cos \alpha \sin \beta \sin \gamma_{V}\right) \\
+Y \cos \gamma_{V}-Z \sin \gamma_{V}-m g \cos \theta \\
-m V \cos \theta \frac{d \psi_{V}}{d t}=P\left(\sin \alpha \sin \gamma_{V}\right. \\
\left.-\cos \alpha \sin \beta \cos \gamma_{V}\right)+Y \sin \gamma_{V}+Z \cos \gamma_{V} \\
\frac{d x}{d t}=V \cos \theta \cos \psi_{V} \\
\frac{d y}{d t}=V \sin \theta \\
\frac{d z}{d t}=-V \cos \theta \sin \psi_{V} \\
\frac{d m}{d t}=-m_{c} \\
\alpha=-\frac{m_{z}^{\delta_{z}}}{m_{z}^{\alpha}} \delta_{z} \\
\beta=-\frac{m_{y}^{\delta_{y}}}{m_{y}^{\beta}} \delta_{y} \\
\varepsilon_{1}=0 \\
\varepsilon_{2}=0 \\
\varepsilon_{3}=0 \\
\varepsilon_{4}=0
\end{array}\right.
$$

where, $t$ is time, $m$ is rocket mass, $V$ is rocket velocity, $\theta$ is flight path angle, $\psi_{V}$ is flight path deflection angle, $P$ is thrust, $X$ is drag, $Y$ is lift, $Z$ is lateral force, $\alpha$ is angle of attack, $\beta$ is angle of sideslip, $\gamma_{V}$ is slop angle of velocity, $x, y, z$ are position coordinates, $m_{c}$ is the mass variation, $g$ is the acceleration of gravity, $\delta_{y}, \delta_{z}$ are rudder deflection angle and elevator angle, $m_{y}^{\delta_{y}}, m_{z}^{\delta_{z}}, m_{y}^{\beta}, m_{z}^{\alpha}$ are rudder control efficiency, elevator control efficiency, yaw static stability derivative, pitch static stability derivative, $\varepsilon_{1}, \varepsilon_{2}, \varepsilon_{3}, \varepsilon_{4}$ are control equations.

\subsection{Power and guidance scheme}

The rocket adopts solid rocket motor, and the thrust scheme is

$$
P= \begin{cases}P_{1} & 0<t \leq t_{1} \\ P_{2} & t_{1}<t \leq t_{2} \\ 0 & t>t_{2}\end{cases}
$$

In [8] a biased proportional guidance law with terminal angular constraint was given

$$
a_{y c}=K V_{m} \dot{q}_{z}+V_{m} \frac{\theta_{f}-\theta}{t_{g o}}-K V_{m} \frac{\theta_{f}-q_{z}}{t_{g o}}
$$

where, $K$ is proportional Coefficient, time to go $t_{g o}=r / V_{m}, \theta_{f}$ is flight path angle at hit, $q_{z}$ is line of sight angle.

The longitudinal flight program of the rocket is: the initial flight path angle is $\theta_{0}$, when $0<t \leq t_{1}$, the rocket is uncontrolled; when $t_{1}<t \leq t_{2}$, the rocket climbs according to the pitching angle command $\vartheta_{c}$; when $t_{2}<t \leq t_{3}$, the rocket is uncontrolled; when $t>t_{3}$ and the missile-target distance $r \geq r_{e}$, the rocket glides at a fixed angle of attack, and the angle of attack command is $\alpha_{c}$; when $r<r_{e}$, the rocket uses the proportional guidance law shown in (3). The lateral flight program of the rocket is: when $y \geq y_{c}$, the rocket flies without control; when $y<y_{c}$, the rocket uses proportional guidance law to fly to the target, and the guidance instruction is $a_{z c}=K_{z} V_{m} \dot{q}_{y}$, where $K_{z}$ is proportional guidance coefficient. The inclined channel flight program of the rocket is: when the rocket is launched, the slope angle command is $\gamma_{c}$.

\section{Optimization model and solution of multiple rockets cooperative attack}

\subsection{Optimization model of cooperative attack}

In order to achieve the cooperative attack of multiple rockets, different launch points are selected to control the attack time of rockets, and the cooperative attack problem is transformed into the distribution of launch points with a given attack time. By solving the optimization model, the distribution of launch points under the same attack time is obtained, and the distribution curve of launch points under the given attack time is fitted. If the attack time interval of the rockets is given, the appropriate launch position can be selected through the distribution curve to achieve cooperative attack.

Let the coordinates of the rocket launch point in the ground coordinate system be $\left(x_{0}, y_{0}, z_{0}\right)$, where $y_{0}=0$, and take $x_{0}, z_{0}$ as the design variable, then the expression of the design variable is

$$
\boldsymbol{X}=\left[x_{1}, x_{2}\right]^{T}=\left[x_{0}, z_{0}\right]
$$

If the attack time of rocket launched by $\left(x_{0}, y_{0}, z_{0}\right)$ is $t$, the objective function of the minimum attack time optimization model is

$$
\min F_{1}(X)=t
$$

The objective function of the maximum attack time optimization model is 


$$
\max F_{2}(X)=t
$$

The objective function of the optimization model of rocket attacking target with expected attack time $\bar{t}$ is

$$
\min F_{3}(\boldsymbol{X})=|t-\bar{t}|
$$

Equations (5) to (7) are solved several times under the constraint conditions, and a set of coordinates of the launch point under the corresponding conditions are obtained. The distribution curve of the launch point can be obtained by fitting the coordinate points.

The constraints of design variables are

$$
\left\{\begin{array}{l}
\boldsymbol{X}_{\text {min }}=\left[x_{0 \min }, z_{0 \min }\right]=[-10000 m,-10000 m] \\
\boldsymbol{X}_{\text {max }}=\left[x_{0 \text { max }}, z_{0 \text { max }}\right]=[10000 m, 10000 m]
\end{array}\right.
$$

Therefore, the feasible region of the optimization model is defined as

$$
\boldsymbol{\Omega}=\left[\boldsymbol{X}_{\min }, \boldsymbol{X}_{\max }\right]
$$

The angle between velocity vector and longitudinal axis of the body is defined as the actual angle of attack of rocket in three-dimensional space

$$
\chi=\arccos (\cos \alpha \cos \beta)
$$

The process and terminal constraints that need to be satisfied in the course of rocket flight are

$$
g_{i}(\boldsymbol{X}) \leq 0, i=1,2,3, \cdots, 6
$$

where

$$
\left\{\begin{array}{l}
g_{1}(\boldsymbol{X})=|\chi|-\chi_{m} \\
g_{2}(\boldsymbol{X})=\theta_{f c}-\left|\theta_{f}\right| \\
g_{3}(\boldsymbol{X})=\left|a_{y}\right|-a_{y m} \\
g_{4}(\boldsymbol{X})=\left|a_{z}\right|-a_{z m} \\
g_{5}(\boldsymbol{X})=V_{\min }-V_{f} \\
g_{6}(\boldsymbol{X})=V_{f}-V_{\max }
\end{array}\right.
$$

where, $\chi_{m}, a_{y m}, a_{z m}$ are maximum values of corresponding parameters, $\theta_{f c}$ is desired falling angle, $V_{\min }, V_{\max }$ are minimum and maximum velocity.

The optimal mathematical models of the minimum attack time, the maximum attack time and the expected attack time of the rockets are shown in (13) to (15).

$$
\begin{array}{ll}
\min & F_{1}=F_{1}(\boldsymbol{X}) \\
\text { s.t. } & g_{i}(\boldsymbol{X}) \leq 0, i=1,2,3, \cdots, 6 \\
& \boldsymbol{X} \in \boldsymbol{\Omega} \\
\max & F_{2}=F_{2}(\boldsymbol{X}) \\
\text { s.t. } & g_{i}(\boldsymbol{X}) \leq 0, i=1,2,3, \cdots, 6 \\
& \boldsymbol{X} \in \boldsymbol{\Omega}
\end{array}
$$

$$
\begin{array}{ll}
\min & F_{3}=F_{3}(\boldsymbol{X}) \\
\text { s.t. } & g_{i}(\boldsymbol{X}) \leq 0, i=1,2,3, \cdots, 6 \\
& \boldsymbol{X} \in \boldsymbol{\Omega}
\end{array}
$$

\subsection{Optimization model solution based on cultural algorithm}

Cultural algorithm is a two-level evolutionary system based on knowledge, which contains two evolutionary spaces, one is belief space composed of experience and knowledge acquired in the process of evolution, the other is population space composed of specific individuals, through evolutionary operation and performance evaluation to carry out its own iterative solution ${ }^{[9,10]}$. Cultural algorithm has been widely used in the field of multi-objective optimization. The framework and flow chart of cultural algorithm are shown in Fig. 1 and Fig. 2 respectively.

The flow of cultural algorithm to solve the problem of optimization of rocket attack time is as follows:

(1) Initialize the population space: Within the range of design variables, the initial population $\mathbf{P}_{0}$ with the scale of $\mathrm{n}$ is randomly generated, i.e. $\mathrm{n}$ groups of rocket launch point coordinates. Then, the $\mathrm{n}$ groups of coordinates generated are respectively substituted into the rocket's trajectory model to obtain the attack time of the rocket. The fitness value $\mathbf{F}$ is calculated according to the target function.

(2) Initialize the knowledge space: The optimal value in population $\mathbf{P}_{0}$ is selected as the initial value of situation knowledge; the lower limit $1_{0}$ and the upper limit $\mathbf{u}_{0}$ are set as $\mathbf{X}_{\min }$ and $\mathbf{X}_{\max }$ in (8) according to the upper and lower limits of design variables, and the evaluation value $\mathbf{L}_{0}$ corresponding to the lower limit $\mathbf{1}_{0}$ and the evaluation value $\mathbf{U}_{0}$ corresponding to the upper limit $\mathbf{u}_{0}$ are initialized as $+\infty$.

(3) Update the population space: According to the cultural algorithm, the iteration step and evolution direction of the design variables of the $t+1$ generation are determined, and $n$ new launch point coordinates are generated, that is, the new group $\mathbf{P}_{t+1}$. The corresponding attack time is obtained by substituting $\mathbf{n}$ new launch point coordinates into the rocket trajectory model, and the fitness value of the individual population is calculated according to the objective function of the optimization model.

(4) Update the knowledge space: According to the cultural algorithm to update the situation knowledge of the knowledge space, the optimal solution $s^{t+1}$ of the first $t+1$ generation is obtained; the normative knowledge of the knowledge space is updated, that is, the upper limit 1 and the lower limit $\mathbf{u}$ of the design variables and the corresponding evaluation values $\mathbf{L}$ and $\mathbf{U}$.

(5) Judge whether the termination conditions are met. If not, go back to step (3).

(6) At the end, the optimal solution is output.

According to the optimization model established in Sec 3.1, the coordinates of launch point in launch 
coordinate system under different attack time can be obtained by using the above methods.

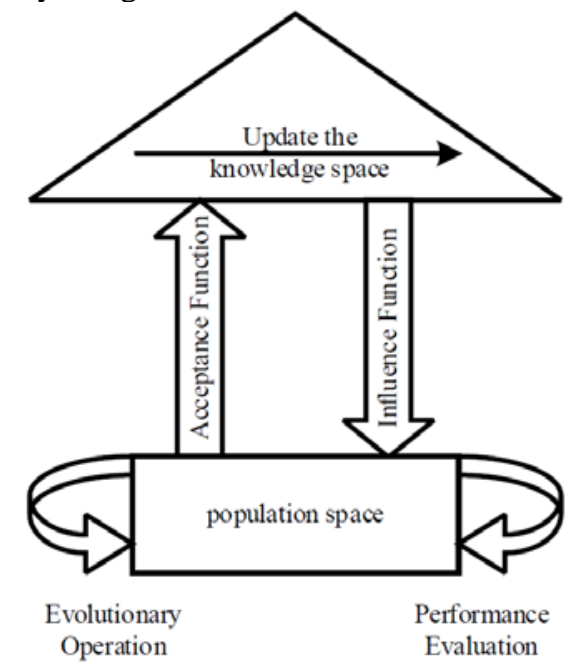

Figure 1. Basic framework of cultural algorithm.

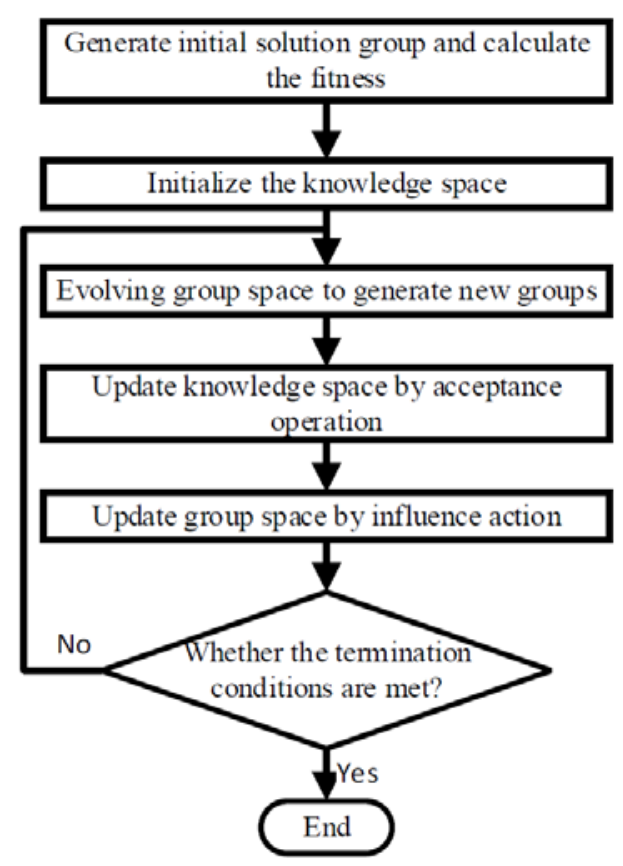

Figure 2. Flow chart of cultural algorithm.

\section{Cooperative strategy of multiple rockets}

In order to achieve multiple rockets attacking the target in a certain order, it can be achieved by setting the position of the rocket launching unit. The specific process is as follows:

(1) According to the solution method given in Sec. 3.2, $m$ sets of launch point coordinates corresponding to the minimum attack time $t_{\min }$ and $m$ sets of launch point coordinates corresponding to the maximum attack time $t_{\max }$ are obtained respectively.

(2) In the rocket attack time range $\left[t_{\min }, t_{\max }\right]$, the expected attack time of the rocket is $\bar{t}_{i}=t_{\min }+i \Delta t, i=1,2, \cdots, n$. Using the same solution method, the coordinates of $m$ launch points corresponding to the expected attack time $\bar{t}_{i}$ are obtained respectively.

(3) Let $\overline{\boldsymbol{T}}=\left[t_{\min }, \bar{t}_{1}, \bar{t}_{2}, \cdots, \bar{t}_{n}, t_{\max }\right]$, use the least square method to fit the coordinates of $m$ group launch points corresponding to each attack time in $\bar{T}$. The distribution curve of launch points under attack time $T_{i}$ is recorded as $S_{i}$, and its expression is recorded as $x_{i}=a_{i} z_{i}^{2}+b_{i} z_{i}+c_{i}, z_{i} \in\left[z_{i \min }, z_{i \max }\right]$.

(4) Select the launch point coordinates according to the actual combat tasks, and consider the cooperative attack of $\mathrm{w}$ rockets on a fixed target. The selection methods of launch points for different cooperative strategies are as follows:

Suppose that multiple rockets attack the target at the same time, as shown in Fig. 3, the cooperative strategy is

$a$. If the attack time $T_{e}$ is equal to the attack time $\bar{T}_{j}$ corresponding to a certain launch point distribution curve $S_{j}$ in (3): Select $S_{j}$ as the launch point distribution curve $S_{e}$; if the attack time $T_{e}$ is not equal to any element in $\overline{\boldsymbol{T}}$, then find the attack time $T_{k}$ in $\overline{\boldsymbol{T}}$ to make it meet $T_{k}<T_{e}<T_{k+1}$, and the analytical expression of the launch point distribution curve $S_{e}$ corresponding to the attack time $T_{e}$ is

$$
x_{e}=x_{k+1}+\frac{T_{k+1}-T_{e}}{\Delta t}\left(x_{k}-x_{k+1}\right)
$$

The analytical formula of $S_{e}$ can be sorted as

$$
\begin{gathered}
x_{e}=a_{e} z_{e}^{2}+b_{e} z_{e}+c_{e} \\
z_{e} \in\left[\max \left(z_{k \min }, z_{k+1 \text { max }}\right), \min \left(z_{k \min }, z_{k+1 \text { max }}\right)\right]
\end{gathered}
$$

where,

$$
\left\{\begin{array}{l}
a_{e}=a_{k+1}+\frac{\left(T_{k+1}-T_{e}\right)\left(a_{k}-a_{k+1}\right)}{\Delta t} \\
b_{e}=b_{k+1}+\frac{\left(T_{k+1}-T_{e}\right)\left(b_{k}-b_{k+1}\right)}{\Delta t} \\
c_{e}=c_{k+1}+\frac{\left(T_{k+1}-T_{e}\right)\left(c_{k}-c_{k+1}\right)}{\Delta t}
\end{array}\right.
$$

The curve $S_{e}$ domain is divided into $w-1$ equal parts, so that the $z$ coordinate of the launch point of the rocket is taken as the point of $w$ - 1 section of the domain, and then the coordinates of the launch point are determined according to the fitting formula.

Suppose that multiple rockets attack the target in order, as shown in Fig. 4, the cooperative strategy is

$b$. If the attack time $T_{e}^{1}, T_{e}^{2}, \cdots, T_{e}^{w}$ of multiple rockets attacking the target in sequence is equal to $\bar{T}_{j}, \bar{T}_{j+1}, \cdots, \bar{T}_{j+w-1}$ in $\overline{\boldsymbol{T}}:$ the launching points of $w$ rockets $\left\{M_{1}, M_{2}, \cdots, M_{w}\right\}$ are respectively located at $S_{j}, S_{j+1}, \cdots, S_{j+w-1}$, where $M_{1}, M_{w}$ is respectively located 
at the endpoint, and the launching point of the ith rocket $M_{i}$ is located at the $i-(w-1)$ th equinox of curve $S_{j+i-1}$. Among them, the point of $w-1$ section of curve $S_{j+i-1}$ are sorted from small to large according to $z$ coordinate. Only the situation that the attack interval is $\Delta t$ is given here, if the expected attack time difference is an integral multiple of $\Delta t$, the principle is the same and will not be repeated.

$c$. If the attack time of multiple rockets attacking the target in sequence is arbitrary $T_{e}^{1}, T_{e}^{2}, \cdots, T_{e}^{w}$ : Firstly, according to the strategy $a$, obtain the distribution curve $S_{e}^{1}$ and $S_{e}^{w}$ of the launch points whose attack time is $T_{e}^{1}$ and $T_{e}^{w}$ respectively; then connect the diagonal ends of $S_{e}^{1}$ and $S_{e}^{w}$ to obtain the straight line $S$ (as shown in Fig. 4); finally, divide the straight line $S$ into $w-1$ equal parts, and select the equal diversion points as the launch points of other rockets. If the given attack time difference is not equal, we can take a point on the straight line $S$ according to the proportion of attack time difference.

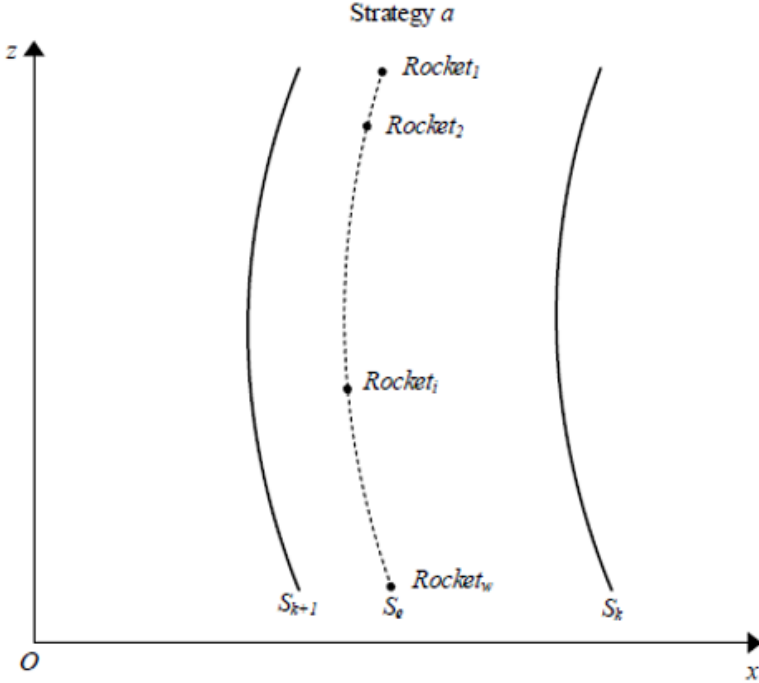

Figure 3. Cooperative strategy for multiple rockets attacking targets at the same time.

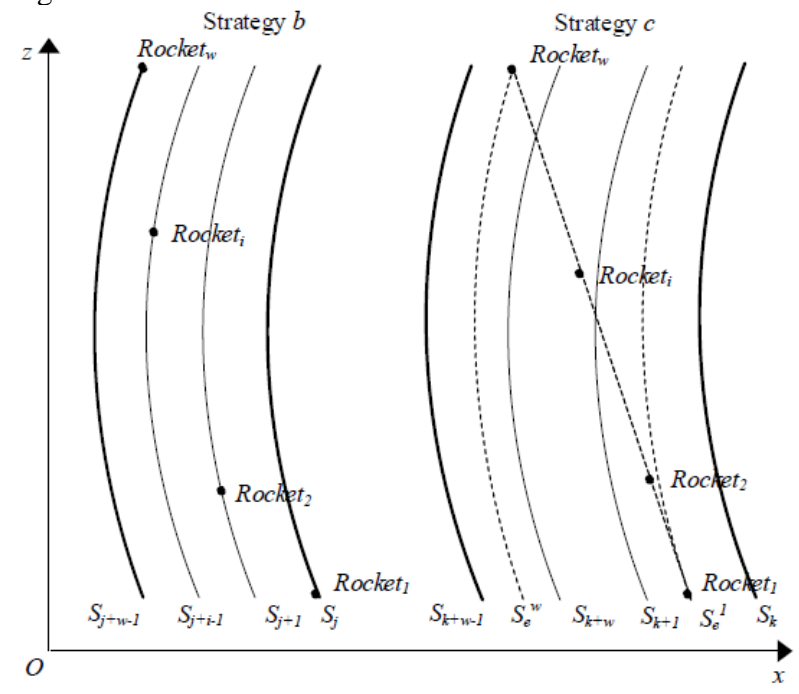

Figure 4. Cooperative strategy of multiple rockets attacking targets in sequence.

\section{Simulation results and analysis}

Based on the rocket motion model established in Sec. 2.1 and the power and guidance scheme in Sec. 2.2, the minimum and maximum attack time optimization models are solved by using the method in Sec. 3.2. See Table 1 for simulation parameter values.

Table 1. Simulation parameter values.

\begin{tabular}{|c|c|}
\hline Variable name & Value \\
\hline$t_{1}$ & $5 s$ \\
\hline$t_{2}$ & $22 \mathrm{~s}$ \\
\hline$t_{3}$ & $200 \mathrm{~s}$ \\
\hline$P_{1}$ & $120 \mathrm{kN}$ \\
\hline$P_{2}$ & $55 \mathrm{kN}$ \\
\hline$\theta_{0}$ & $50^{\circ}$ \\
\hline$\vartheta_{c}$ & $45^{\circ}$ \\
\hline$r_{e}$ & $30 \mathrm{~km}$ \\
\hline$\alpha_{c}$ & $8^{\circ}$ \\
\hline$y_{c}$ & $20 \mathrm{~km}$ \\
\hline$\gamma_{c}$ & 0 \\
\hline K & 5 \\
\hline$K_{z}$ & 3 \\
\hline$\chi_{m}$ & $10^{\circ}$ \\
\hline$a_{y m}$ & $10^{\circ}$ \\
\hline$a_{z m}$ & $10^{\circ}$ \\
\hline$\theta_{f c}$ & $80^{\circ}$ \\
\hline$V_{\min }$ & $600 \mathrm{~m} / \mathrm{s}$ \\
\hline$V_{\max }$ & $900 \mathrm{~m} / \mathrm{s}$ \\
\hline
\end{tabular}

The population size is set to 20 , and the termination generation of the algorithm is set to 100 . The minimum attack time of the rockets is 280s, and the maximum attack time is $302.5 \mathrm{~s}$. Set the expected attack time $\bar{t}=282,284,286, \cdots, 300$, calculate 50 times for each optimized model of the expected attack time, and get the distribution of launch points as shown in Fig. 5. 


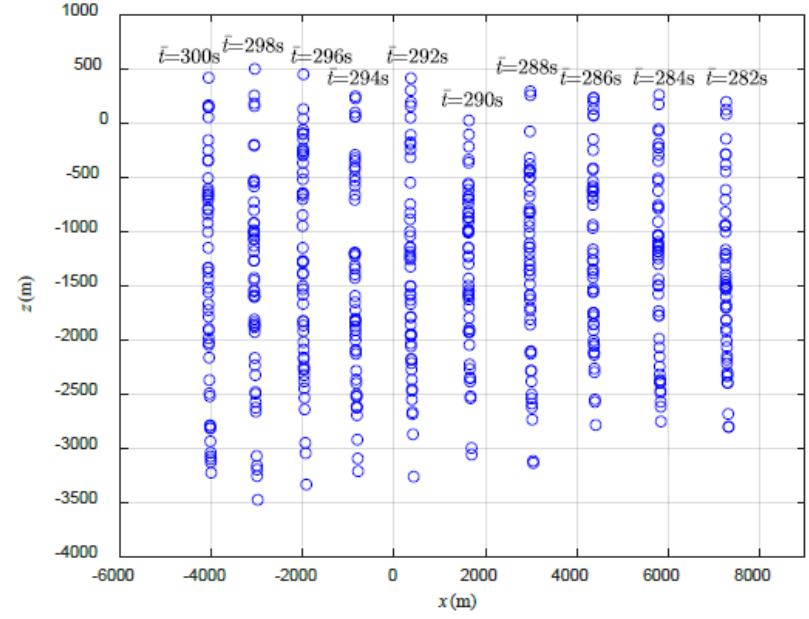

Figure 5. Distribution of launch points under different expected attack times.

The distribution curves of the launching points are fitted, as shown in Table 2.

It can be seen from Table 2 that when the least square method is used for the quadratic polynomial fitting of the optimization results, the complex correlation coefficient approaches to 1 , indicating that the fitting method has high precision, and the fitting results are shown in Fig. 6. To verify the fitting results, select 100 points evenly on each curve for simulation, and the attack time error is shown in Fig. 7.
In order to verify the multiple missiles cooperative strategy proposed in Sec. 4, four rockets are assumed to attack the target cooperatively, and three cooperative strategies proposed in this paper are used for simulation.

1) Four rockets are required to hit the target at the same time, and the attack time is 291s. According to strategy $a$, the distribution curve of launch point with attack time of 291s is deduced as follows

$$
\begin{gathered}
x_{e 291}=14.81 z_{e 291}^{2}+25.37 z_{e 291}+1000 \\
z_{e 291} \in[-3.061 \mathrm{~km}, 0.022 \mathrm{~km}]
\end{gathered}
$$

The launching positions of the four rockets are shown in Fig. 8 (marked with "॰" in the figure), and the simulation results are shown in Fig. 9 to Fig. 11.

It can be seen from Fig. 8 to Fig. 11 that the four rockets meet the end velocity constraint, and the time of convergence to $30 \mathrm{~m}$ is 290.98s, 290.99s, 290.98s, 290.99s, and the maximum attack time difference is $0.01 \mathrm{~s}$.

2) Four rockets are required to hit the target in a certain order, and the attack time is 290s, 292s, 294s and 296s respectively. According to strategy $b$, select the launch location as shown in Fig. 12 (marked with "०" in the figure), and the simulation results are shown in Fig. 13 to Fig. 15.

\begin{tabular}{|c|c|c|c|}
\hline $\begin{array}{l}\text { Attack } \\
\text { time(s) }\end{array}$ & Fitting formula(m) & $\begin{array}{c}\text { Range of independent } \\
\text { variable }\end{array}$ & $\begin{array}{c}\text { Complex } \\
\text { correlation } \\
\text { coefficient }\left(R^{2}\right) \\
\end{array}$ \\
\hline 280 & $x_{1}=8.72 z_{1}^{2}+3.79 z_{1}+8816$ & {$[-2.499 \mathrm{~km}, 0.005 \mathrm{~km}]$} & 0.9189 \\
\hline 282 & $x_{2}=18.41 z_{2}^{2}+31.35 z_{2}+7274$ & {$[-2.806 \mathrm{~km}, 0.193 \mathrm{~km}]$} & 0.9830 \\
\hline 284 & $x_{3}=17.98 z_{3}^{2}+30.26 z_{3}+5798$ & {$[-2.756 \mathrm{~km}, 0.258 \mathrm{~km}]$} & 0.9831 \\
\hline 286 & $x_{4}=15.97 z_{4}^{2}+26.77 z_{4}+4366$ & {$[-2.789 \mathrm{~km}, 0.232 \mathrm{~km}]$} & 0.9798 \\
\hline 288 & $x_{5}=15.54 z_{5}^{2}+25.74 z_{5}+2978$ & {$[-3.139 \mathrm{~km}, 0.291 \mathrm{~km}]$} & 0.9893 \\
\hline 290 & $x_{6}=15.90 z_{6}^{2}+28.32 z_{6}+1640$ & {$[-3.061 \mathrm{~km}, 0.022 \mathrm{~km}]$} & 0.9883 \\
\hline 292 & $x_{7}=13.71 z_{7}^{2}+22.41 z_{7}+360$ & {$[-3.264 \mathrm{~km}, 0.412 \mathrm{~km}]$} & 0.9798 \\
\hline 294 & $x_{8}=12.98 z_{8}^{2}+21.24 z_{8}-853$ & {$[-3.215 \mathrm{~km}, 0.245 \mathrm{~km}]$} & 0.9906 \\
\hline 296 & $x_{9}=12.90 z_{9}^{2}+21.72 z_{9}-1994$ & {$[-3.337 \mathrm{~km}, 0.447 \mathrm{~km}]$} & 0.9906 \\
\hline 298 & $x_{10}=11.60 z_{10}^{2}+18.38 z_{10}-3065$ & {$[-3.478 \mathrm{~km}, 0.498 \mathrm{~km}]$} & 0.9937 \\
\hline 300 & $x_{11}=10.98 z_{11}^{2}+16.73 z_{11}-4072$ & {$[-3.229 \mathrm{~km}, 0.417 \mathrm{~km}]$} & 0.9919 \\
\hline 302.5 & $x_{12}=25.35 z_{12}^{2}+70.08 z_{12}-5192$ & {$[-3.414 \mathrm{~km},-0.122 \mathrm{~km}]$} & 0.9283 \\
\hline
\end{tabular}

Table 2. Fitting results of distribution curve of emission points. 


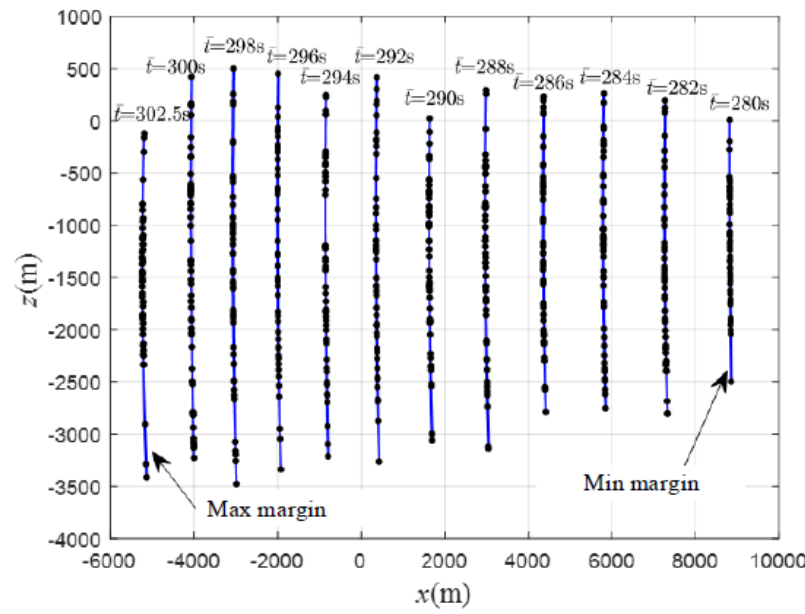

Figure 6. Distribution curve of launch points with different attack time.
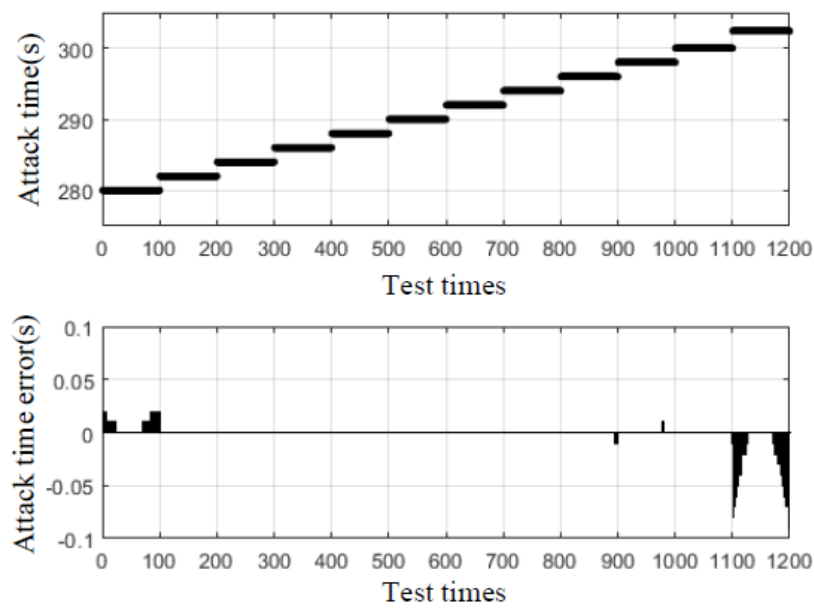

Figure 7. Attack time error.

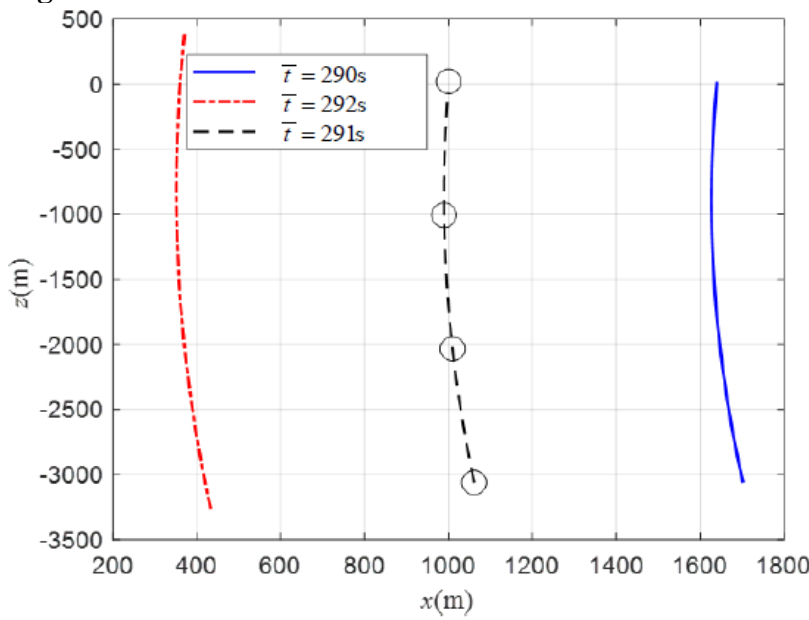

Figure 8. Launch point location of cooperative strategy $a$.

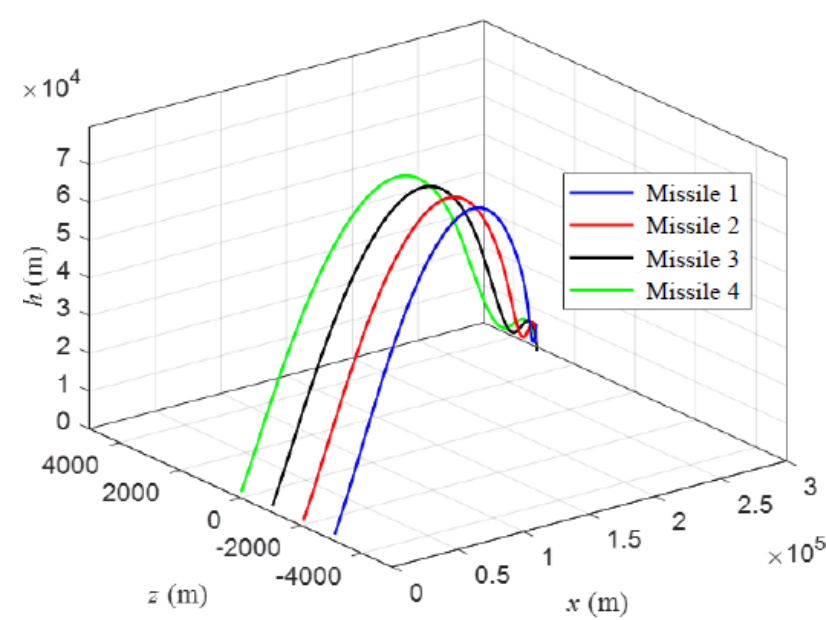

Figure 9. Three dimensional trajectory diagram of cooperative strategy $a$.

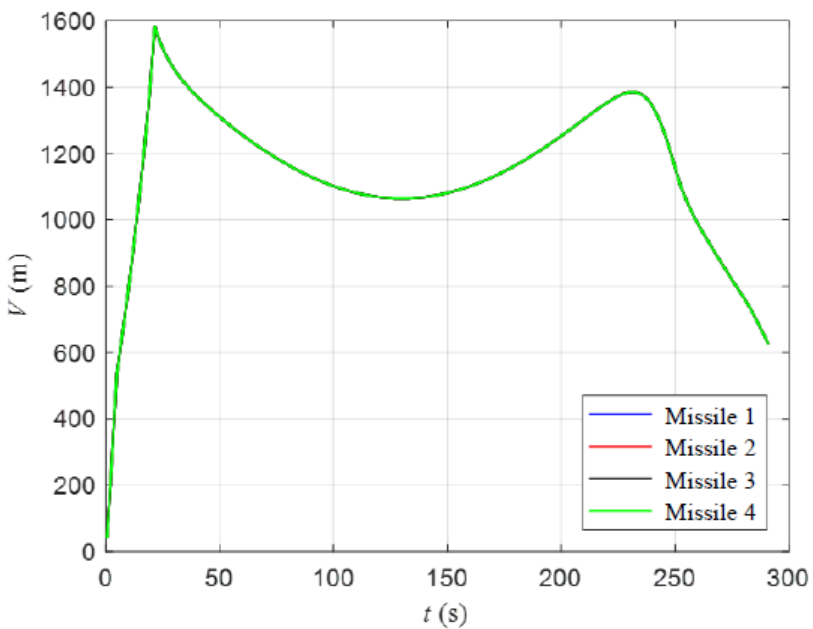

Figure 10. Speed curve of collaborative strategy $a$.

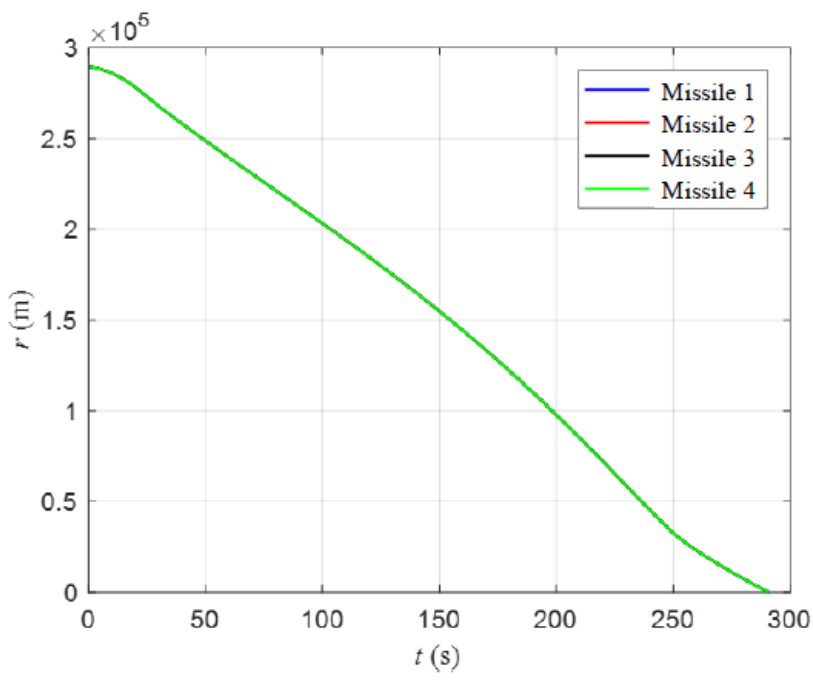

Figure 11. Missile target distance curve of cooperative strategy 


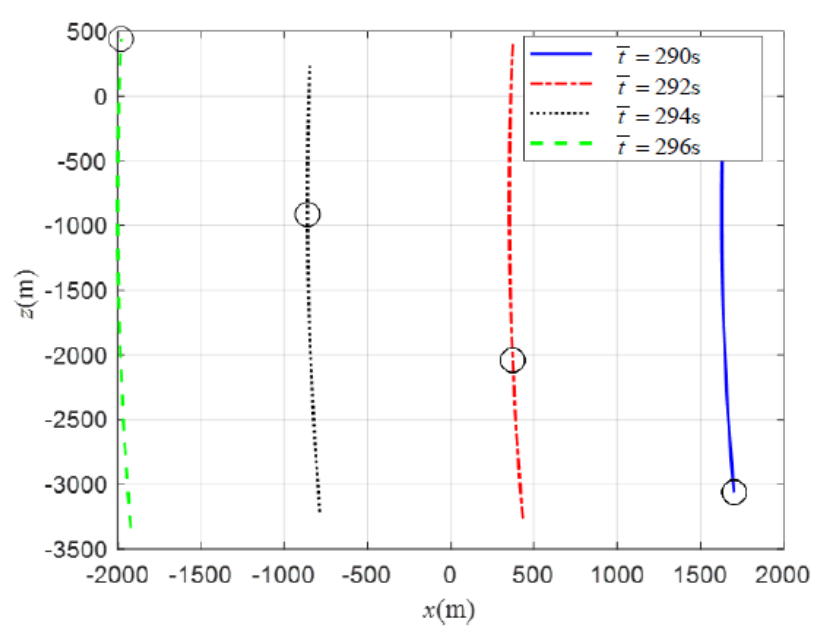

Figure 12. Launch point location of cooperative strategy $b$.

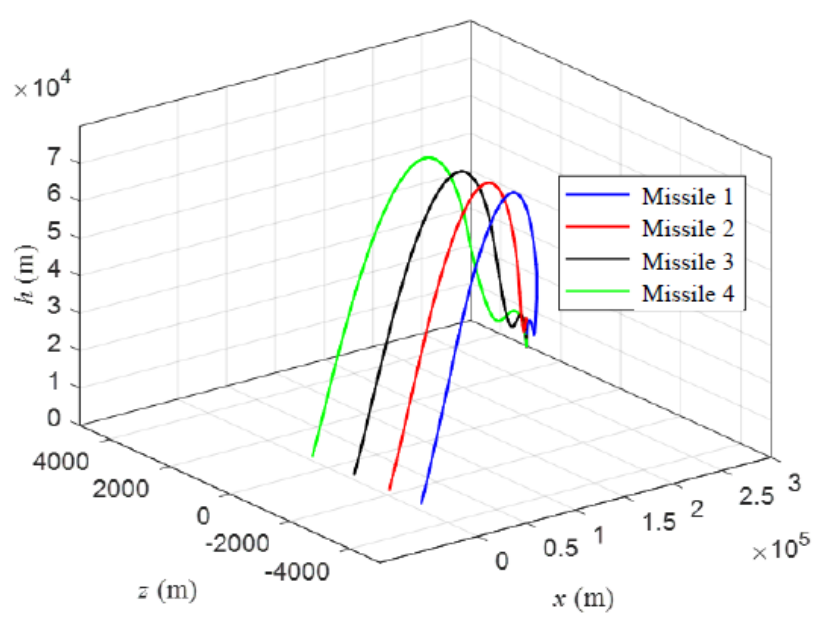

Figure 13. Three dimensional trajectory diagram of cooperative strategy $b$.

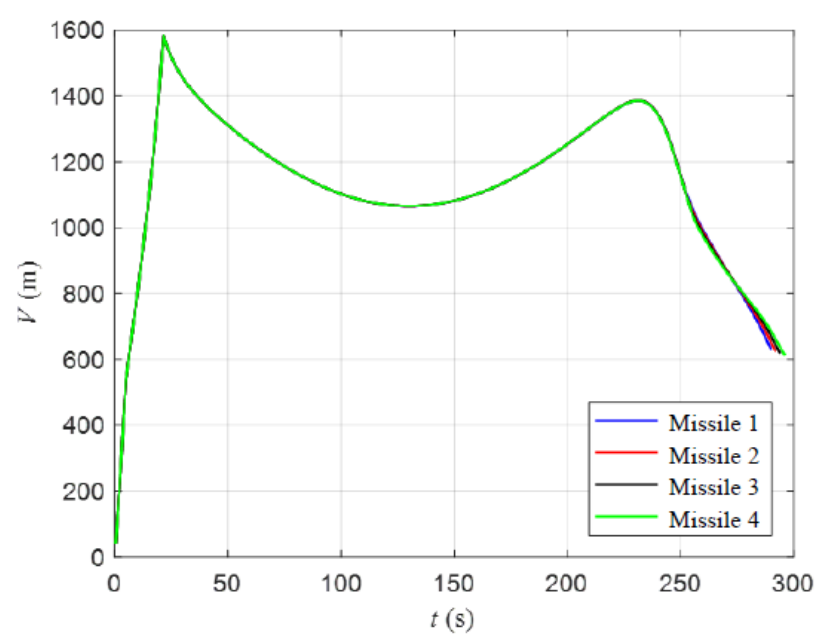

Figure 14. Speed curve of collaborative strategy $b$.

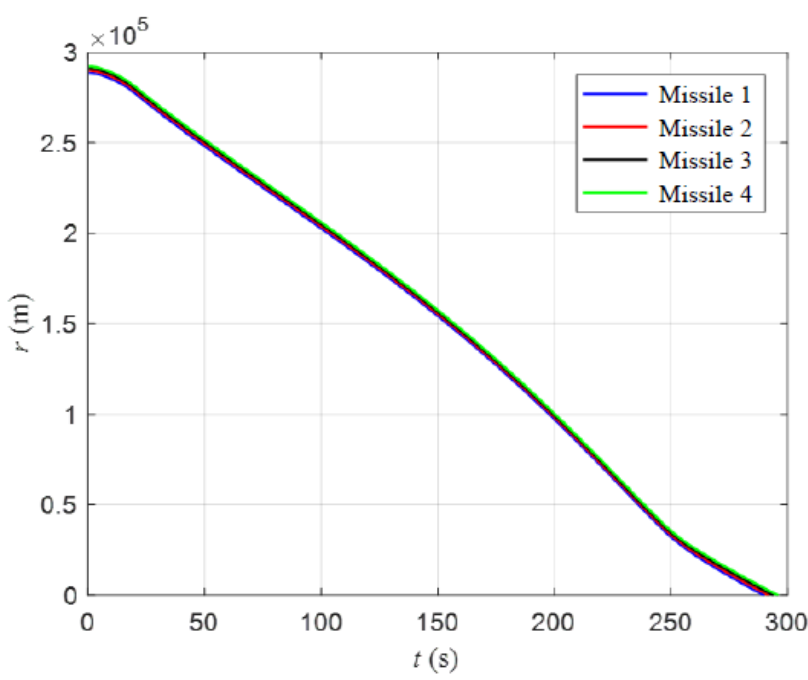

Figure 15. Missile target distance curve of cooperative strategy

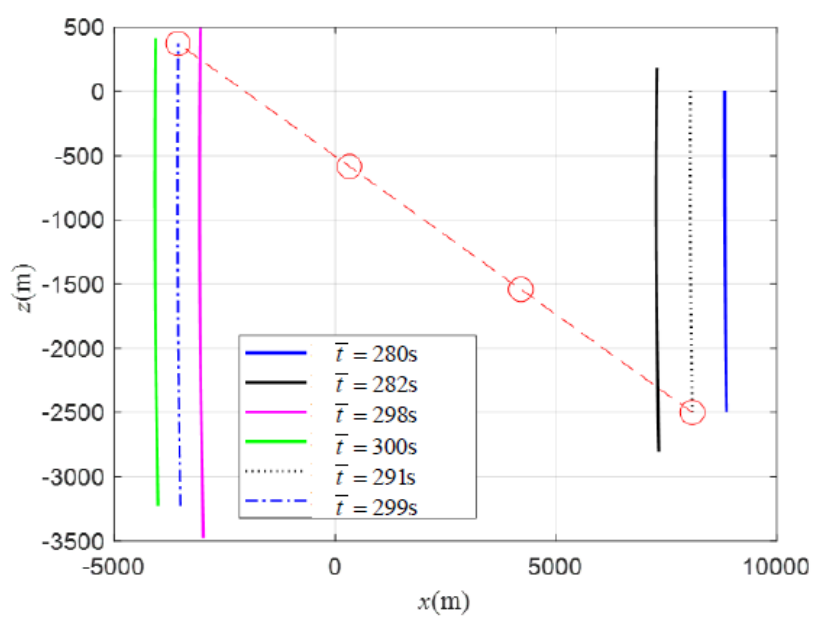

Figure 16. Launch point location of cooperative strategy $c$.

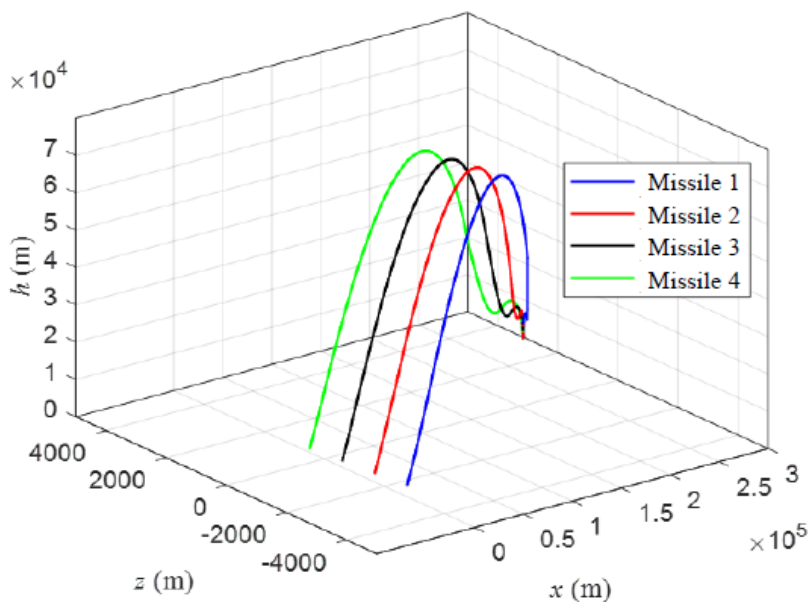

Figure 17. Three dimensional trajectory diagram of cooperative strategy $c$. 


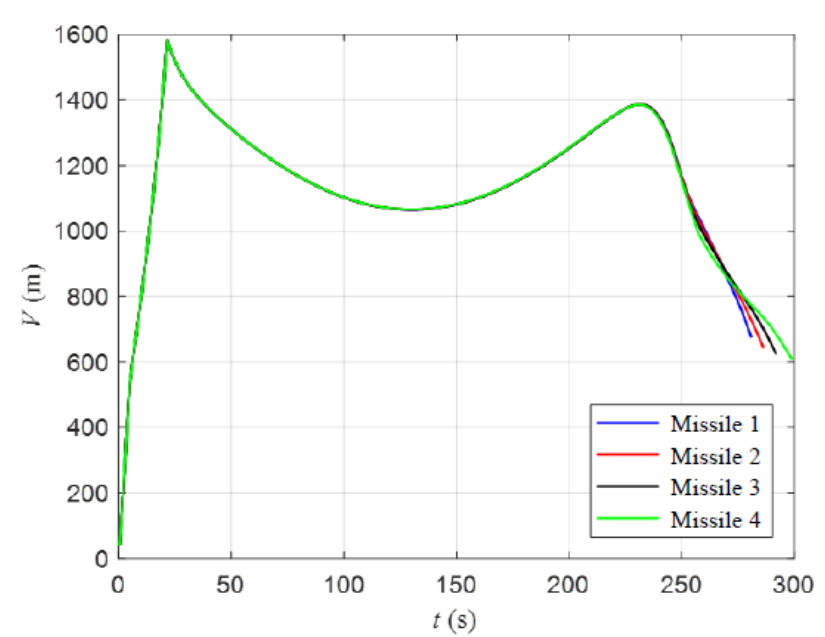

Figure 18. Speed curve of collaborative strategy c.

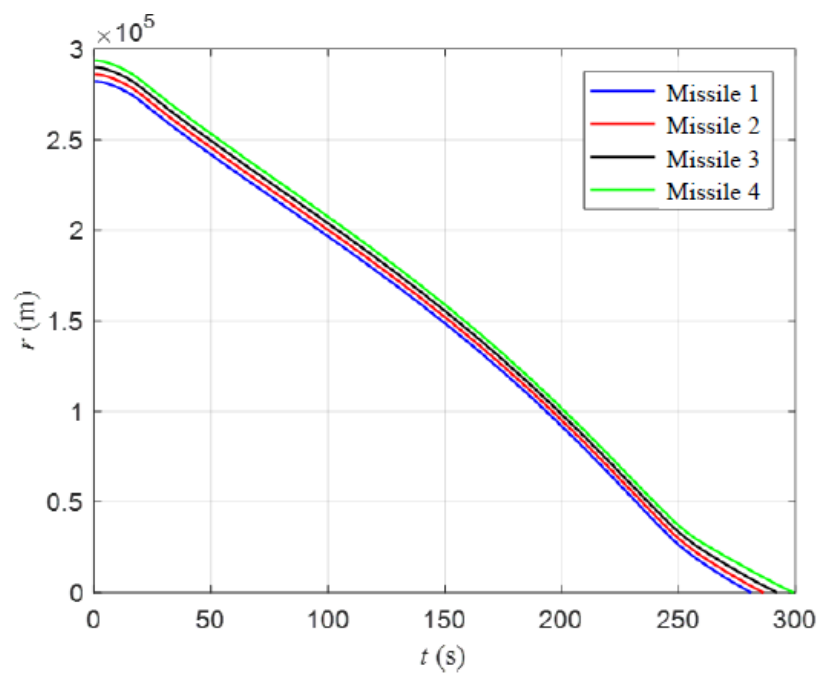

Figure 19. Missile target distance curve of cooperative strategy c.

It can be seen from Fig. 12 to Fig. 15 that the four rockets meet the end velocity constraint, and the time of convergence to 30m is 290s, 292s, 294s and 295.99s.

3) four rockets are required to hit the target at $6 \mathrm{~s}$ interval, and the attack time is 281s, 287s, 293s and 299s respectively. According to strategy $c$, the distribution curve of launch points with attack time of 281s and 299s is deduced as follows

$$
\begin{gathered}
x_{e 281}=13.57 z_{e 281}^{2}+17.57 z_{e 281}+8045 \\
z_{e 281} \in[-2.499 \mathrm{~km}, 0.005 \mathrm{~km}] \\
x_{e 299}=11.29 z_{e 299}^{2}+17.56 z_{e 299}-3569 \\
z_{e 299} \in[-3.229 \mathrm{~km}, 0.417 \mathrm{~km}]
\end{gathered}
$$

recorded as $S_{281}$ and $S_{299}$ respectively. The launch positions of the four rockets are shown in Fig. 16 (marked with "○" in the figure), and the simulation results are shown in Fig. 17 to Fig. 19.
It can be seen from Fig. 16 to Fig. 19 that the four rockets meet the end velocity constraint. The time of convergence to $30 \mathrm{~m}$ is $280.98 \mathrm{~s}, 286.22 \mathrm{~s}, 292.05 \mathrm{~s}$ and 298.98s. The attack time interval is 5.24s, 5.83s and 6.93s, respectively. Compared with the expected 6s interval, the error is within $1 \mathrm{~s}$.

\section{Conclusions}

In this paper, the trajectory design and optimization of the rockets are studied, the motion model of the rocket's center of mass is established, and the power and guidance scheme are designed. In order to solve the problem of multiple rockets cooperative attack, a multiple rockets cooperative attack scheme based on different launch points is proposed. The cooperative attack problem of rockets is transformed into the optimization problem of launch point. The optimization problem is solved by cultural algorithm, and the distribution curve of launch point with different attack time is fitted. Based on the distribution curve of launch points, the cooperative combat strategy of multiple rockets is proposed to attack targets simultaneously or in a specified order. The next problems need to be solved are the elastic vibration, the coupling between the aerodynamic force and the control system, and the aerodynamic heat of the rocket when it enters the atmosphere at a high speed from the highest point of the trajectory.

\section{References}

1. L. Y. Xie, R. Liao, X. Q. Wang, Command Control \& Simulation, 4, 30 (2009)

2. Y. Huang, D. H. Wang, H. C. Wang, J. Hu, W. H. Xie, Journal of Projectiles, Rockets, Missiles and Guidance, 5, 127 (2012)

3. Y. Huang, J. D. Huang, J. You, Journal of Projectiles, Rockets, Missiles and Guidance, 5, 6 (2018)

4. L. D. Cheng, Y. Jiang, Y. C. Niu, Journal of Ballistics, 2, 19 (2018)

5. D. Cho, H. J. Kim, M. J. Tahk, Journal of Guidance Control and Dynamics, 1, 61 (2016)

6. S. Gutman Journal of Guidance, Control, and Dynamics, 8, 2107 (2017)

7. Y. An, K. Park and C. Ryoo, 9th International Conference on Mechanical and Aerospace Engineering, 362 (2018)

8. F. Gao, S. J. Tang, J. Shi, J. Guo, Transactions of Beijing Institute of Technology, 3, 277 (2014)

9. H. Y. Huang, X. S. Gu, M. D. Liu, ACTA AUTOMATICA SINICA, 10, 1115 (2007)

10. Z. J. Qi, M. D. Liu, COMPUTER TECHNOLOGY AND DEVELOPMENT, 5, 126 (2008) 